\title{
An Unexpected Dual-Emissive Luminogen: Tunable Aggregation-Induced Emission with Cyan-White-Red Colors, Stable Inherent Chirality, and Enhanced Chiroptical Property
}

\author{
Xinyu Zhang, ${ }^{a}$ Huiqing Liu, ${ }^{a}$ Guilin Zhuang, ${ }^{b}$ Shangfeng Yang, ${ }^{* a}$ Pingwu Du ${ }^{\text {*a }}$ \\ ${ }^{a}$ Hefei National Laboratory for Physical Sciences at the Microscale, CAS Key Laboratory of Materials for Energy Conversion, Department of Materials Science and \\ Engineering, $i \mathrm{ChEM}$, University of Science and Technology of China, 96 Jinzhai Road, Hefei, Anhui Province, 230026, China. \\ ${ }^{\mathrm{b}}$ College of Chemical Engineering, Zhejiang University of Technology, 18, Chaowang Road, Hangzhou, Zhejiang Province, 310032 , China
}

\begin{abstract}
Herein we report a novel chiral bismacrocycle with unexpected dual emission and tunable aggregation-induced emission colors. A facile four-step synthesis strategy is developed to construct this rigid bismacrocycle, $(1,4)[8]$ cycloparaphenylenophane (SCPP[8]), which possesses a 1,2,4,5-tetraphenylbenzene core locked by two intersecting polyphenylene-based macrocycles. The luminescent behavior of SCPP $[8]$ shows the unique characteristics of both aggregation-caused quenching effect and aggregation-induced emission (AIE) effect, inducing remarkable redshift emission including near white-light emission. SCPP[8] is configurationally stable and possesses a novel shape-persistent bismacrocycle scaffold with a high strain energy (up to $127.83 \mathrm{kcal} / \mathrm{mol}$ ). In addition, SCPP[8] displays enhanced circularly polarized luminescence properties due to AIE effect.
\end{abstract}

Aggregation-induced emission (AIE) phenomenon has enormous potential applications in biological probes, chemical sensors, and optoelectronic materials. ${ }^{1-3}$ The concept of AIE has received much attention since B. Z. Tang et al. described it in 2001 and great progress has been made since then. ${ }^{4-9}$ In many traditional systems, luminophores often emit strongly in their dilute solutions but meet with varying degrees of aggregation-caused quenching (ACQ) effect when they are aggregated or clustered. In contrast, non-emissive luminogens are induced to emit by the aggregate formation in the AIE systems. ${ }^{6}$ Therefore, AIE luminogens (AIEgens) show remarkable advantages over typical ACQ molecules, especially in their aggregate and solid states. ${ }^{10-11}$ However, in the literature, materials with both AIE and ACQ effects that can emit both in the solution and aggregated state are rarely reported. The solution and solid dual-emissive luminogens can exactly fill the gap between ACQ and AIE materials, providing many interesting properties such as stimuli-responsive fluorescence and white-light emission. ${ }^{12-16}$ Most of the AIE systems have been identified on the basis of intensity turn-on at a specific wavelength. If AIE sensors have tunable emission colors based on the manipulation of aggregation, they can endow a dimension with the discriminative power and allow naked-eye visualization. ${ }^{17}$ Therefore, the development of novel dual-emissive luminogens is highly desired.

Typical AIEgens, such as hexaphenylsilole (HPS) and tetraphenylethene (TPE), have been well studied (Scheme 1). ${ }^{18-20}$ In solution, the dynamic rotations of the phenyl rings in TPE as a relaxation channel for the excitons to dissipate. Upon aggregate formation, such motion is restricted due to the restricted intramolecular rotation (RIR) and intermolecular $\pi-\pi$ stacking interaction derived from the highly twisted molecular conformation. ${ }^{21-22}$ By immobilization of propeller-shaped AIE molecules with various functional groups, new AIEgens will form and exhibit many different properties. ${ }^{23-25}$ This design approach also takes advantage of the emission features in the AIE-active core, providing new systems with novel physical properties and applications. ${ }^{12,26-27}$ Moreover, when attaching chiral moieties to the propeller-shaped structures, many new applications are reported in chiral sensors and chiroptical materials. ${ }^{28-31} 1,2,4,5-$ tetraphenylbenzene $(1,2,4,5-\mathrm{TPB})$ is another kind of AIEgens that also features a propeller-shaped structure (Scheme 1), ${ }^{32}$ which is an attractive building block for the construction of chiral molecules with novel AIE properties. In sharp contrast to linear paraphenylenes, cycloparaphenylenes (CPPs) are radial $\pi$-conjugated macrocycles that compose of para-connected benzene rings with unique properties. ${ }^{33-36}$ By bridging two CPP-based macrocycles in a central benzene, a chiral bismacrocycle molecule with AIE-active $1,2,4,5$-TPB core can be formed. Although we recently synthesized a [10]CPP-based bismacrocycle, ${ }^{37}$ its poor solubility and large ringsize prevent us to resolve the enantiomers and study the chiral properties. The synthesis of a smaller bismacrocycle is even more attractive due to better solubility for enantiomeric resolution and excellent size-dependent properties for AIE study. So far, no such an example was reported.

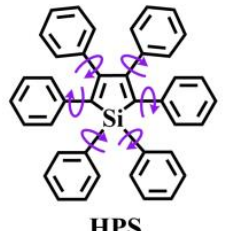

HPS

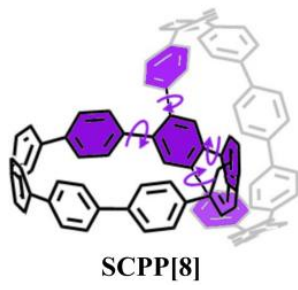

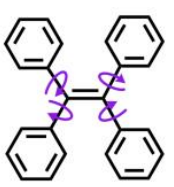

TPE

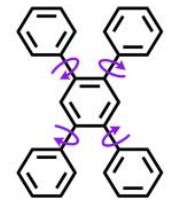

$1,2,4,5-T P B$
This work

Aggregation-Induced Emission

Tunable cyan-white-red Emission

Stable inherent chirality

Enhanced CPL

Scheme 1. Top: Typical examples of structural motifs of AIEgens. Bottom: Structure of SCPP[8] molecule.

Herein, by immobilization of the AIE-active 1,2,4,5-TPB unit with rigid para-phenylene units, a novel inherently chiral and unexpected dual-emissive bismacrocycle was synthesized via a facile four-step synthesis. SCPP[8] can emit efficiently both in solution (cyan) and in aggregation (red). The fluorescence emission maximum of SCPP [8] in the aggregated state showed a remarkable redshift $(102 \mathrm{~nm})$ from that in the dilute solution. More intriguingly, 
multicolor cyan-white-red photoluminescence can be tuned by manipulating the aggregation, realizing near white-light emission with a CIE coordinate at about $(0.33,0.37)$. SCPP $[8]$ possesses a novel scaffold of nonracemizable chiral structure with enhanced chiroptical properties due to AIE effect. The physical, (chir)optical, and inherently chiral properties were further investigated.

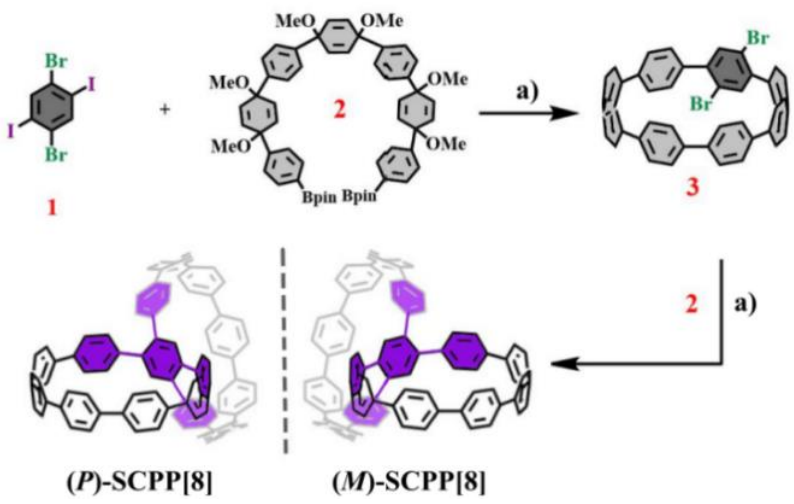

Scheme 2. Synthesis procedure for inherently chiral SCPP[8] with 1,2,4,5TPB core. a) (i) $\mathrm{Pd}\left(\mathrm{PPh}_{3}\right)_{4}, \mathrm{KOH}, \mathrm{THF} / \mathrm{H}_{2} \mathrm{O}, 2$ days at $73{ }^{\circ} \mathrm{C}$; (ii) $\mathrm{H}_{2} \mathrm{SnCl}_{4}$, THF, RT.

The synthesis of small chiral bismacrocycle with a 1,2,4,5TPB core poses a great challenge because of the increasing strain energy in a small curved molecule. Despite many attempts, our previous approach ${ }^{37}$ using an -OTf functional group was unsuccessful for SCPP[8]. Next, we chose a 1,2,4,5-halogensubstituted benzene to react with a $\mathrm{C}$-shaped synthon to produce a macrocyclic precursor, followed by reductive aromatization to generate a bisubstituted macrocycle (Scheme 2). Since the bisubstituted benzene can rotate rapidly, the enantiomers cannot be separated. Under a similar coupling reaction, the bisubstituted CPP macrocycle was further reacted with the $\mathrm{C}$-shaped synthon to lock the central benzene and fix its rotation, resulting in an inherently chiral bismacrocycle with a 1,2,4,5-TPB core locked by rigid polyphenylene units.

The successful synthesis of SCPP[8] can be supported by different characterization techniques, including high-resolution mass spectrometry (HR-MS), ${ }^{1} \mathrm{H}$ NMR, ${ }^{13} \mathrm{C}$ NMR, 2D ${ }^{1} \mathrm{H}-{ }^{1} \mathrm{H}$ COSY NMR, 2D NOESY NMR, 2D (H, C)-HSQC NMR, and 2D (H, C)-HMBC NMR (Figures S1-S10). The molecular weight of SCPP[8] was determined by MALDI-TOF MS spectrometry. The main peak at $\mathrm{m} / z, 1138.4593$ was observed (calculated for $\mathrm{C}_{90} \mathrm{H}_{58}$ $\left.[M]^{+}: 1138.4538\right)$, which matched well with the calculated data. Besides, the crystalline solid can be obtained by slow diffusion of methanol into a SCPP[8] solution in dichloromethane (Figure 2b). Unfortunately, these crystals are extremely fragile, and the large crystals tend to break immediately once exposed to air. Therefore, so far the crystal structure of SCPP[8] cannot be resolved.

SCPP [8] molecule is calculated to has exceptionally high strain energy up to $127 \mathrm{kcal} / \mathrm{mol}$ at the B3LYP/6-31G(d) level (Figure 1a). This bismacrocycle molecule is highly strained, and its ring strain is even much larger than the very small nanoring [5]CPP $(119 \mathrm{kcal} / \mathrm{mol}) .{ }^{38} \mathrm{We}$ concluded that the structural factors contribute to the unexpectedly large strain energy in SCPP[8]. Compared with the individual phenylene, the central benzene bridged by two carbon nanorings has much larger resistance to twisting. In addition, the limited conformational freedom in SCPP[8] leads to significant multiple repulsive interactions between adjacent phenylene fragments. The geometrical optimization results indicated that SCPP[8] features chiral $C_{2}$ symmetry, and the twist angle in the bridging phenyl is $\sim 19.55^{\circ}$. The frontier molecular orbitals of [8]CPP and SCPP[8] are shown in Figures $1 \mathrm{~b}$ and 1c, respectively. The energy levels of the occupied molecular orbitals of SCPP[8] move to higher positions than those of [8]CPP, while the unoccupied molecular orbitals move to lower positions than those of [8]CPP. Therefore, the SCPP[8] achieves a smaller HOMO-LUMO gap of $\sim 2.780 \mathrm{eV}$ compared to [8]CPP.

(a)

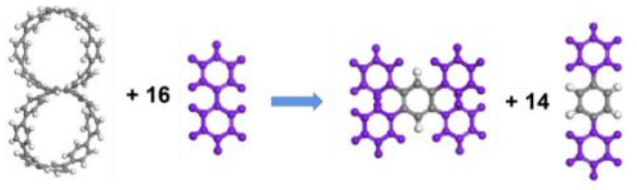

(b)

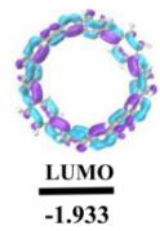

(c)
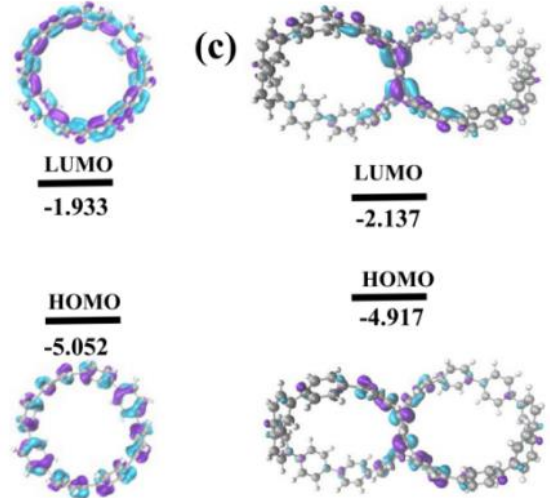

Figure 1. (a) Homodesmotic equation for the calculation of strain energy for SCPP[8]. (b) LUMO and HOMO of [8]CPP. (c) LUMO and HOMO of SCPP $[8]$.

The photophysical properties of SCPP[8] were further studied by UV-Vis absorption spectroscopy, fluorescence spectroscopy, and time-resolved fluorescence decay (Figure 2). A single macrocyclic [8]CPP was used as a reference compound for comparison. The lower symmetry of $\mathbf{S C P P}[8]\left(C_{2}\right)$ in comparison with the circular CPPs $\left(D_{2}\right)$ results in a more complex pattern of electronic transitions, as determined in time-dependent density functional theory (TD-DFT) calculations. The absorption band of SCPP [8] was in the region between 265 and $436 \mathrm{~nm}$, and the maximum absorption peak $\left(\lambda_{\max }\right)$ appears at $368 \mathrm{~nm}$ (Figure 2a). The extinction coefficients reach up to $1.22 \times 10^{5} \mathrm{M}^{-1} \cdot \mathrm{cm}^{-1}$. To identify the difference of optical properties, density functional theory calculations were performed by using Gaussian 16 software. In accordance with the aforementioned decrease in the HOMOLUMO gap, the absorption spectrum of SCPP[8] showed an obvious redshift compared with [8]CPP. Based on the calculated results, an excellent correlation between the calculated and experimental absorption spectra was observed. The three main absorption peaks can be attributed to the transition of HOMO- $2 \rightarrow$ LUMO for the peak at $368 \mathrm{~nm}, \mathrm{HOMO} \rightarrow \mathrm{LUMO}+2$ for the peak at $346 \mathrm{~nm}$, and HOMO-1 $\rightarrow$ LUMO+5 for the peak at $305 \mathrm{~nm}$. In addition, the observed absorption peak of [8]CPP at $340 \mathrm{~nm}$ can be assigned to the transition of HOMO-1 $\rightarrow$ LUMO and HOMO $\rightarrow$ LUMO+1. The redshift effect of the absorption spectrum in SCPP[8] compared to [8]CPP was ascribed to the shift of the frontier molecular orbitals.

The steady-state fluorescence emission spectrum of SCPP[8] was further measured (Figure 2c). SCPP[8] and [8]CPP showed cyan and yellow fluorescence in tetrahydrofuran (THF) with fluorescence maxima at $475 \mathrm{~nm}$ and $533 \mathrm{~nm}$, respectively. The fluorescence quantum yield $\left(\Phi_{\mathrm{F}}\right)$ was determined to be $\sim 3 \%$ using anthracene in ethanol as the reference $\left(\Phi_{\mathrm{F}}=30 \%\right)$, and the yield is lower than that of $[8] \mathrm{CPP}\left(\Phi_{\mathrm{F}}=10 \%\right)$, which probably results from its high proportion of nonradiative decay in the low-energy emission region. 
The fluorescence lifetime $\left(\tau_{\mathrm{s}}\right)$ of $\mathbf{S C P P}[8]$ in THF was measured by time-resolved fluorescence decay using the timeresolved photoluminescence (TRPL) technique. The emission lifetime of SCPP[8] was determined to be $\tau=4.23 \mathrm{~ns}$ at $475 \mathrm{~nm}$ by mono-exponential decay fitting when excited at $390 \mathrm{~nm}$ (Figure 2d).

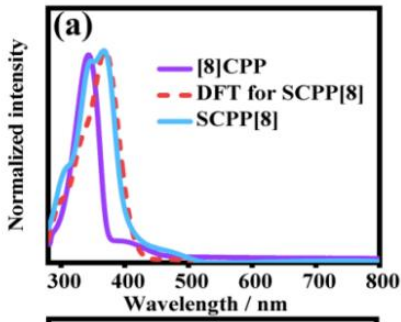

(b)
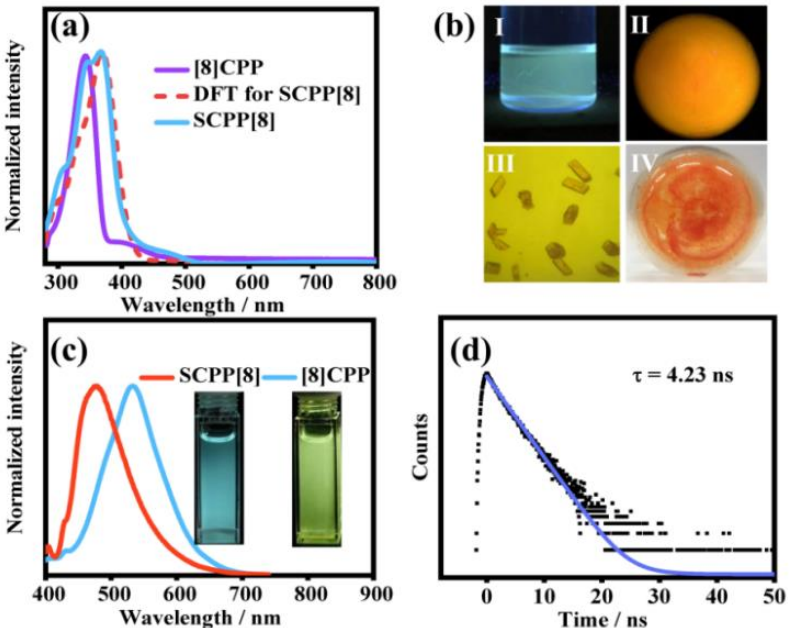

Figure 2. (a) UV-Vis absorption spectra of SCPP[8] (blue) and [8]CPP (purple) in THF. Simulated curve of UV spectrum for SCPP[8] (dotted line). (b) Photos of SCPP[8] in THF solution (I), as powder (II) under $365 \mathrm{~nm}$ UV light, as crystal (III), and as powder (IV) under daylight. (c) Fluorescence spectra of SCPP[8] (red) and [8]CPP (blue) in THF. Insert: [8]CPP and SCPP[8] in THF solution under $365 \mathrm{~nm}$ UV light. (d) Emission lifetime for SCPP[8].

Traditional organic luminescent materials can emit efficiently either in dilute solution or in the aggregated state. However, an interesting phenomenon was found when we studied the luminescence behavior of SCPP[8]. Upon excitation with $365 \mathrm{~nm}$ UV light, SCPP[8] in THF solution emitted cyan fluorescence, while the powder sample exhibited an orange fluorescence. The powder and crystal of SCPP [8] were orange under daylight (Figure 2b). All these observations indicated that SCPP[8] has different emission properties upon aggregation with possible AIE effect. The luminescent properties of the SCPP[8] were further investigated in $\mathrm{H}_{2} \mathrm{O} / \mathrm{THF}$ mixtures with different water fractions $\left(f_{\mathrm{w}}\right)$, in which THF is a good solvent and water is a poor solvent. The fluorescence intensity of SCPP[8] maximized at $\sim 475 \mathrm{~nm}$ decreased as the water fraction was increased, showing an obvious quenching effect due to aggregation. An interesting phenomenon was observed at $f_{\mathrm{w}}>60$ vol \%. A new emission band appeared at $\sim 577 \mathrm{~nm}$ and the fluorescence intensity was further enhanced when a higher content of water was added, which could be ascribed to the obvious AIE effect (Figure 3a). Meanwhile, the maximum emission wavelength was redshifted by $102 \mathrm{~nm}$, from $475 \mathrm{~nm}$ to $577 \mathrm{~nm}$. For comparison, when [8]CPP was dissolved in THF, the dilute solution showed strong yellow luminescence. With the gradual increase in the fraction of water, the emission became weakened and no any AIE effect was observed. At $f_{\mathrm{w}}=80 \mathrm{vol} \%$ and $f_{\mathrm{w}}=90$ vol $\%$, the emission was significantly quenched due to severe aggregate formation, indicating a typical ACQ effect (Figure S12 and Figure $3 d)$. For comparison, the emissive properties of our previously reported [10]CPP-based bismacrocycle SCPP[10] with larger rings were also investigated in $\mathrm{H}_{2} \mathrm{O}$ /THF mixtures (Figure $\mathrm{S} 11$ ). However, SCPP[10] only showed some AIE effect with no dual-emissive property and no such significant redshift in emission.

The emission color changes of SCPP[8] from cyan to red were recorded in aqueous THF with $f_{\mathrm{w}}=0-99$ vol $\%$ under $365 \mathrm{~nm}$ UVlight (Figure 3c). Intriguingly, a near white-light emission was observed at $f_{\mathrm{w}}=60 \mathrm{vol} \%$. In the literature, most organic whitelight emitters depend on the combination of several components that emit different colors of light. ${ }^{39-41}$ Compared with multicomponent emitters, single-molecule systems with whitelight emission have excellent stability, reproducibility, and a simplified fabrication process. ${ }^{42-44}$ The photoluminescence spectra of SCPP[8] at $f_{\mathrm{w}}=0-99$ vol $\%$ were obtained, and their corresponding luminescent color coordinates were calculated and plotted in the CIE 1931 chromaticity diagram (Figure 3b). The near white-light emission has a CIE coordinate of about $(0.33,0.37)$. Therefore, SCPP[8] can be considered as a potential candidate for organic white-light emitting materials.
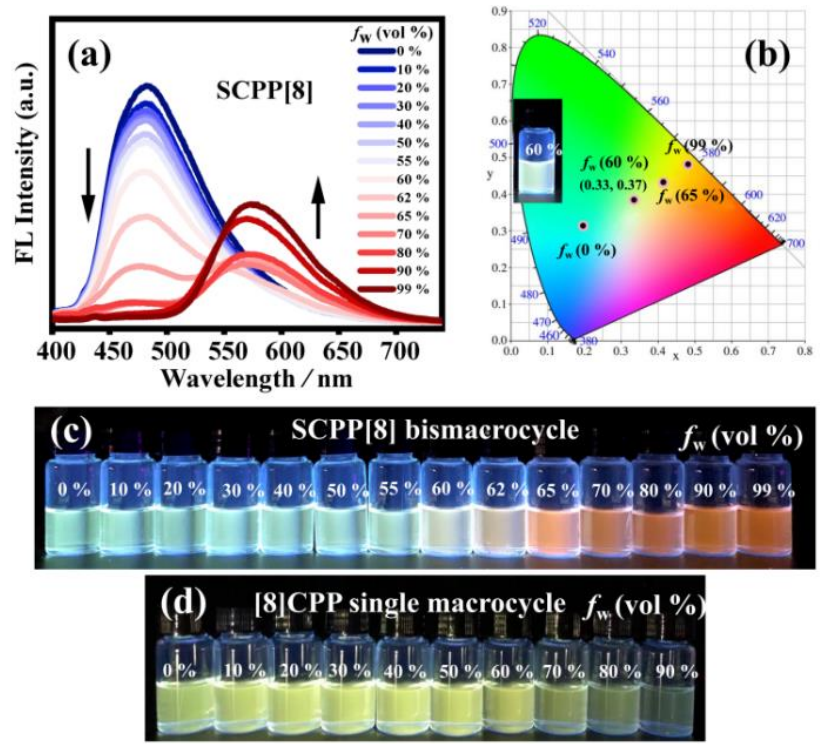

Figure 3. (a) Fluorescence spectra of SCPP[8] in $\mathrm{THF} / \mathrm{H}_{2} \mathrm{O}$ mixtures with different volume fractions of $\mathrm{H}_{2} \mathrm{O}\left(f_{\mathrm{w}}\right)$. (b) CIE 1931 chromaticity diagram of SCPP[8] in THF/ $/ \mathrm{H}_{2} \mathrm{O}$ mixtures. Insert: The near white-light of SCPP[8] in aqueous THF with $f_{\mathrm{w}}=60 \mathrm{vol} \%$ under $365 \mathrm{~nm}$ UV light. (c) Emission color changes of SCPP[8] from cyan to red in aqueous THF with $f_{\mathrm{w}}=0-99$ vol \% under $365 \mathrm{~nm}$ UV light. (d) Emission change of [8]CPP in aqueous THF with $f_{\mathrm{w}}=0-90 \mathrm{vol} \%$ under $365 \mathrm{~nm}$ UV light.

The present fluorescence behaviors are quite different from those of traditional ACQ or AIE materials. SCPP[8] is an unexpected dual-emissive luminogen that can emit both in the solution and aggregated state. The luminescence behavior of SCPP[8] combines the characteristics of the ACQ effect and AIE effect, inducing tunable emission including near white-light emission. In contrast, the reference compound [8]CPP only shows a typical ACQ effect. Therefore, the unique photophysical properties in SCPP[8] can be attributed to the locked conjugated bismacrocycle structure and the AIE-active 1,2,4,5-TPB core.

The structure of SCPP[8] consists of a twisted benzene ring bridged by polyphenylene units, resulting in a stable and rigid conformation. This excellent conformational stability enables complete enantiomeric resolution and characterizations. After purification by flash column chromatography, the resolution of racemic samples was achieved by a recycling HPLC system. After only two cycles, we succeeded in the isolation of the complete set of two enantiomers $(\boldsymbol{P})$-SCPP $[8]$ and $(\boldsymbol{M})$-SCPP[8]. The extremely narrow peaks in HPLC strongly indicated excellent separation of the two enantiomers, and the molar ratio of the two isomers was determined to be approximately 1:1 (Figure S13). The enantiomeric relationship of the (P)-SCPP[8] and $(\boldsymbol{M})$-SCPP[8] was identified using CD spectroscopy. In Figure $4 a$, the CD spectrum of $(\boldsymbol{M})$ SCPP[8] showed two negative Cotton effects at 299 and $371 \mathrm{~nm}$ and two positive ones at 336 and $467 \mathrm{~nm}$. Being the enantiomer of (M)-SCPP[8], (P)-SCPP[8] gave the expected mirror-imaged CD spectrum, confirming their enantiomeric relationship. The 
magnitude of the CD spectra can be quantified by the dissymmetry factor $\left(g_{\mathrm{CD}}\right)$, which is the ratio of CD intensity to the corresponding absorption. A large $|g \mathrm{CD}|$ of $\sim 0.012$ was obtained for the enantiomers of SCPP[8]. To further elucidate the absolute configuration of $(\boldsymbol{P})$-SCPP $[8]$ and $(\boldsymbol{M})$-SCPP[8], we investigated the theoretical CD spectra on the basis of TD-DFT calculations, as shown in Figure $4 \mathrm{a}$ (dashed plot). The theoretical CD spectra of the resolved enantiomers were in good agreement with the experimental results.
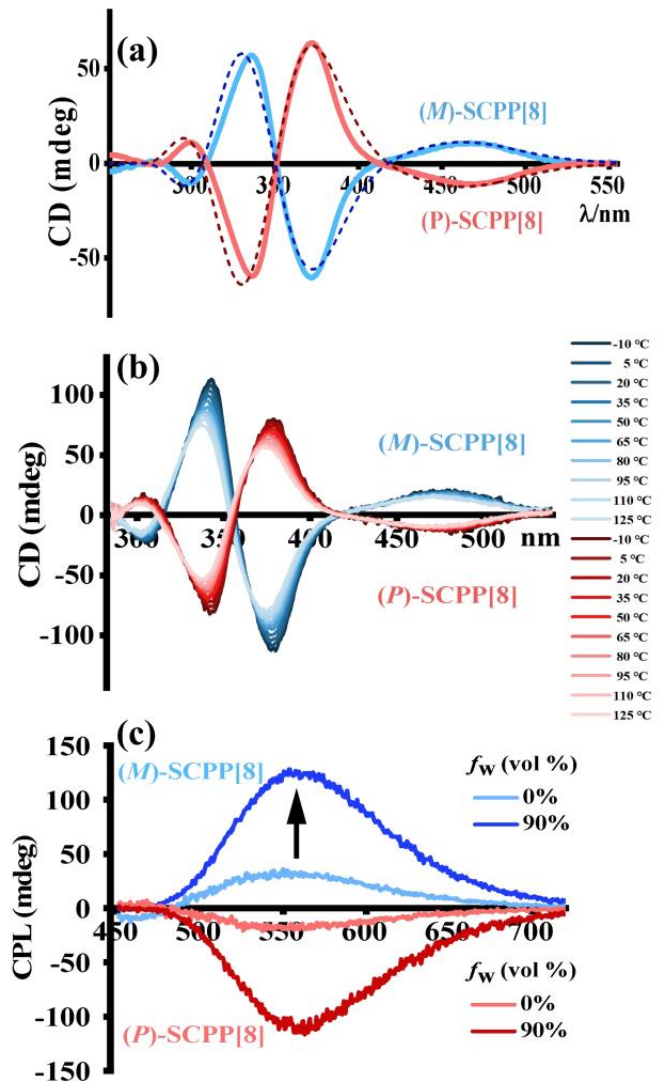

Figure 4. (a) Experimental and theoretical CD spectra for $(\boldsymbol{P})$-SCPP[8] and $(\boldsymbol{M})$-SCPP[8]. The solid lines are the experimental spectra of $(\boldsymbol{M})$-SCPP[8] (blue) and (P)-SCPP[8] (red) in dichloromethane. The dashed lines are theoretical spectra of $(\boldsymbol{M})$-SCPP[8] (dark blue) and $(\boldsymbol{P})$-SCPP[8] (dark red) from TD-DFT calculations. (b) Temperature-dependent CD spectra of $(\boldsymbol{M})$ SCPP [8] (blue) and (P)-SCPP[8] (red) from $-10{ }^{\circ} \mathrm{C}$ to $125{ }^{\circ} \mathrm{C}$ at $15{ }^{\circ} \mathrm{C}$ intervals. (c) CPL spectra of (M)-SCPP[8] (blue) and (P)-SCPP[8] (red) in THF solution and in 90:10 $\mathrm{H}_{2} \mathrm{O} / \mathrm{THF}$ solution. Excited at $355 \mathrm{~nm}$.

To gain insight into the configurational stability of $(\boldsymbol{P})$ SCPP[8] and (M)-SCPP[8], we used temperature-dependent CD spectra to investigate the conformational behavior of these two enantiomers. Chlorobenzene was chosen as the solvent as it allowed access to a wide range of temperatures. To investigate the temperature-dependence of the $\mathrm{CD}$-signals, the samples were heated from $-10{ }^{\circ} \mathrm{C}$ to $125{ }^{\circ} \mathrm{C}$, and $\mathrm{CD}$ spectra were recorded at $15^{\circ} \mathrm{C}$ intervals (Figure $4 \mathrm{~b}$ ). The results clearly showed that optical activity of $(\boldsymbol{P})$-SCPP[8] and (M)-SCPP[8] have no significant change even after heating to $125{ }^{\circ} \mathrm{C}$. The slight change can be attributed to the influence of the solvent at different temperatures, and the change can be recovered by cooling down the solvent (Figure S14). This result showed that the resolved enantiomers of SCPP[8] are configurationally stable, maintaining their optical activity in a wide range of temperatures. These rigid bismacrocycles contain nonracemizable structures, and the geometry can preclude significant loss of enantiomeric purity during further research and application processes. Therefore, the isolation and characterization of SCPP[8] enantiomers shed light on a novel scaffold of nonracemizable chiral bismacrocycles with potential applications as chiroptical material.

The circularly polarized luminescence (CPL) is quantified through the luminescence dissymmetry factor ( $\left.g_{\text {lum }}\right)$, which is defined as the ratio of the difference of the left- and right-circularly polarized light to half of the total luminescence. ${ }^{45} \mathrm{CPL}$-active materials fabricated from AIEgens show much larger dissymmetry factors in the solid state than the molecules in solution. ${ }^{1,23,46} \mathrm{Sig}$ nificantly, $(\boldsymbol{M})$-SCPP[8] and (P)-SCPP[8] exhibited highly enhanced CPL signals in the aggregated state (Figure $4 \mathrm{c}$ ). In a THF solution, the $g_{\text {lum }}$ was $6.9 \times 10^{-3}$ for $(\boldsymbol{M})$-SCPP[8] at $554 \mathrm{~nm}$ and $6.1 \times 10^{-3}$ for $(\boldsymbol{P})$-SCPP[8] at $558 \mathrm{~nm}$. In a $\mathrm{H}_{2} \mathrm{O} / \mathrm{THF}(90: 10$, v/v) solution, the $\left|g_{\text {lum }}\right|$ is $\sim 3$-fold larger than that in solution, which is $1.9 \times 10^{-2}$ for $(\boldsymbol{M})$-SCPP[8] at $562 \mathrm{~nm}$ and $-1.8 \times 10^{-2}$ for $(\boldsymbol{P})$ SCPP[8] at $564 \mathrm{~nm}$. The results showed a high luminescence dissymmetry factor compared with many established CPL-active organic molecules, whose factors are typically in the range of $10^{-3}$ to $10^{-4} \cdot{ }^{45,47}$ Therefore, chiral SCPP[8] molecules have great potential in applications as CPL-active materials.

\section{Conclusion}

In summary, we report the construction of a dual-emissive SCPP [8] bismacrocycle by immobilization of 1,2,4,5-TPB core with two rigid macrocycles. The luminescence behavior of SCPP[8] combines the characteristics of the ACQ effect and AIE effect, inducing tunable emission from cyan to red including near whitelight emission. The enantiomers of $(\boldsymbol{M})$-SCPP[8] and (P)-SCPP[8] are separated by a recycling chiral HPLC, and the high configurational stability of two enantiomers are demonstrated by temperature-dependent CD spectra. Further, $(\boldsymbol{M})$-SCPP[8] and $(\boldsymbol{P})$ SCPP $[8]$ exhibit highly enhanced CPL properties due to AIE effect. $(\boldsymbol{M}) /(\boldsymbol{P})$-SCPP[8] will have potential applications in AIE sensors, white-light emitters, and chiral materials.

\section{ASSOCIATED CONTENT}

\section{Supporting Information}

Additional experimental data include Synthesis details, ${ }^{1} \mathrm{H}$ NMR, ${ }^{13} \mathrm{C}$ NMR, HR-MS, HPLC, and AIE data.

\section{AUTHOR INFORMATION}

\section{Corresponding Author}

Email: $\underline{\text { dupingwu@ustc.edu.cn; } \text { sfyang@ustc.edu.cn }}$ Notes

The authors declare no competing financial interests.

\section{ACKNOWLEDGMENT}

This work was financially supported by the National Key Research and Development Program of China (2017YFA0402800), the National Natural Science Foundation of China (21971229, U1932214).

\section{REFERENCES}

1. Mei, J.; Leung, N. L. C.; Kwok, R. T. K.; Lam, J. W. Y.; Tang, B. Z., Aggregation-Induced Emission: Together We Shine, United We Soar! Chem. Rev. 2015, 115 (21), 11718-11940.

2. Cai, X.; Liu, B., Aggregation-Induced Emission: Recent Advances in Materials and Biomedical Applications. Angew. Chem. Int. Ed. 2020, 59 (25), 9868-9886.

3. Zhao, Z.; Zhang, H.; Lam, J. W. Y.; Tang, B. Z., AggregationInduced Emission: New Vistas at the Aggregate Level. Angew. Chem. Int. Ed. 2020, 59 (25), 9888-9907.

4. Luo, J.; Xie, Z.; Lam, J. W. Y.; Cheng, L.; Chen, H.; Qiu, C.; Kwok, H. S.; Zhan, X.; Liu, Y.; Zhu, D.; Tang, B. Z., 
Aggregation-Induced Emission of 1-Methyl-1, 2, 3, 4, 5pentaphenylsilole. Chem. Commun. 2001, (18), 1740-1741.

5. Banal, J. L.; Zhang, B.; Jones, D. J.; Ghiggino, K. P.; Wong, W. W. H., Emissive Molecular Aggregates and Energy Migration in Luminescent Solar Concentrators. Acc. Chem. Res. 2017, 50 (1), 49-57.

6. Mei, J.; Hong, Y.; Lam, J. W. Y.; Qin, A.; Tang, Y.; Tang, B. Z., Aggregation-Induced Emission: The Whole Is More Brilliant than the Parts. Adv. Mater. 2014, 26 (31), 5429-5479.

7. Li, Q.; Li, Z., The Strong Light-Emission Materials in the Aggregated State: What Happens from a Single Molecule to the Collective Group. Adv. Sci. 2017, 4 (7), 1600484.

8. Xu, S.; Duan, Y.; Liu, B., Precise Molecular Design for HighPerformance Luminogens with Aggregation-Induced Emission. Adv. Mater. 2020, 32 (1), 1903530.

9. Hong, Y.; Lam, J. W. Y.; Tang, B. Z., Aggregation-Induced Emission. Chem. Soc. Rev. 2011, 40 (11), 5361-5388.

10. Ding, D.; Li, K.; Liu, B.; Tang, B. Z., Bioprobes Based on AIE Fluorogens. Acc. Chem. Res. 2013, 46 (11), 2441-2453.

11. Wang, H.; Zhao, E.; Lam, J. W. Y.; Tang, B. Z., AIE Luminogens: Emission Brightened by Aggregation. Mater. Today 2015, 18 (7), 365-377.

12. Lei, S. N.; Xiao, H.; Zeng, Y.; Tung, C. H.; Wu, L. Z.; Cong, H., BowtieArene: A Dual Macrocycle Exhibiting Stimuli-Responsive Fluorescence. Angew. Chem. Int. Ed. 2020, 59 (25), 10059-10065.

13. Li, D.; Hu, W.; Wang, J.; Zhang, Q.; Cao, X. M.; Ma, X.; Tian, H., White-Light Emission from A Single Organic Compound With Unique Self-folded Conformation and Multistimuli Responsiveness. Chem. Sci. 2018, 9 (26), 5709-5715.

14. Chen, G.; Li, W.; Zhou, T.; Peng, Q.; Zhai, D.; Li, H.; Yuan, W. Z.; Zhang, Y.; Tang, B. Z., Conjugation-Induced Rigidity in Twisting Molecules: Filling the Gap Between AggregationCaused Quenching and Aggregation-Induced Emission $A d v$. Mater. 2015, 27 (30), 4496-4501.

15. Wu, H.; Chen, Z.; Chi, W.; Bindra, A. K.; Gu, L.; Qian, C.; Wu, B.; Yue, B.; Liu, G.; Yang, G.; Zhu, L.; Zhao, Y., Structural Engineering of Luminogens with High Emission Efficiency Both in Solution and in the Solid State. Angew. Chem. Int. Ed. 2019, 58 (33), 11419-11423.

16. Wang, G.; Yu, H.; Yang, L.; He, Z.; Zhou, L.; Sun, J.; Gu, X.; Yang, W.; Tang, B. Z., Core-Shell Fluorescent Polymeric Particles with Tunable White Light Emission Based on Aggregation Microenvironment Manipulation. Angew. Chem. Int. Ed. 2021, 60, 25246-25251.

17. Gao, M.; Tang, B. Z., Fluorescent Sensors Based on AggregationInduced Emission: Recent Advances and Perspectives. ACS Sens. 2017, 2 (10), 1382-1399.

18. Hong, Y.; Lam, J. W. Y.; Tang, B. Z., Aggregation-Induced Emission: Phenomenon, Mechanism and Applications. Chem. Commun. 2009, (29), 4332-4353.

19. Hu, R.; Leung, N. L. C.; Tang, B. Z., AIE Macromolecules: Syntheses, Structures and Functionalities. Chem. Soc. Rev. 2014, 43 (13), 4494-4562.

20. Chi, Z.; Zhang, X.; Xu, B.; Zhou, X.; Ma, C.; Zhang, Y.; Liu, S.; $\mathrm{Xu}$, J., Recent Advances in Organic Mechanofluorochromic Materials. Chem. Soc. Rev. 2012, 41 (10), 3878-3896.

21. Leung, N. L. C.; Xie, N.; Yuan, W.; Liu, Y.; Wu, Q.; Peng, Q.; Miao, Q.; Lam, J. W. Y.; Tang, B. Z., Restriction of Intramolecular Motions: The General Mechanism behind Aggregation-Induced Emission. Chem. Eur. J. 2014, 20 (47), 15349-15353.

22. Zhao, Z.; Lam, J. W. Y.; Tang, B. Z., Tetraphenylethene: A Versatile AIE Building Block for the Construction of Efficient Luminescent Materials for Organic Light-Emitting Diodes. $J$. Mater. Chem. 2012, 22 (45), 23726-23740.

23. Xiong, J. B.; Feng, H. T.; Sun, J. P.; Xie, W. Z.; Yang, D.; Liu, M.; Zheng, Y. S., The Fixed Propeller-Like Conformation of Tetraphenylethylene that Reveals Aggregation-Induced Emission Effect, Chiral Recognition, and Enhanced Chiroptical Property. J. Am. Chem. Soc. 2016, 138 (36), 11469-11472.
24. Gao, C.; Seow, J. Y.; Zhang, B.; Hall, C. R.; Tilley, A. J.; White, J. M.; Smith, T. A.; Wong, W. W. H., Tetraphenylethene 9, 10Diphenylanthracene Derivatives-Synthesis and Photophysical Properties. ChemPlusChem 2019, 84 (6), 746-753.

25. Sturala, J.; Etherington, M. K.; Bismillah, A. N.; Higginbotham, H. F.; Trewby, W.; Aguilar, J. A.; Bromley, E. H. C.; Avestro, A. J.; Monkman, A. P.; McGonigal, P. R., Excited-State Aromatic Interactions in the Aggregation-Induced Emission of Molecular Rotors. J. Am. Chem. Soc. 2017, 139 (49), 17882-17889.

26. Li, Y.; Dong, Y.; Cheng, L.; Qin, C.; Nian, H.; Zhang, H.; Yu, Y.; Cao, L., Aggregation-Induced Emission and Light-Harvesting Function of Tetraphenylethene-Based Tetracationic Dicyclophane. J. Am. Chem. Soc. 2019, 141 (21), 8412-8415.

27. Liu, H.; Gu, Y.; Dai, Y.; Wang, K.; Zhang, S.; Chen, G.; Zou, B.; Yang, B., Pressure-Induced Blue-Shifted and Enhanced Emission: A Cooperative Effect between Aggregation-Induced Emission and Energy-Transfer Suppression. J. Am. Chem. Soc. 2020, 142 (3), 1153-1158.

28. Qu, H.; Wang, Y.; Li, Z.; Wang, X.; Fang, H.; Tian, Z.; Cao, X., Molecular Face-Rotating Cube with Emergent Chiral and Fluorescence Properties. J. Am. Chem. Soc. 2017, 139 (50), 18142-18145.

29. Wu, Y.; You, L. H.; Yu, Z. Q.; Wang, J. H.; Meng, Z.; Liu, Y.; Li, X. S.; Fu, K.; Ren, X. K.; Tang, B. Z., Rational Design of Circularly Polarized Luminescent Aggregation-Induced Emission Luminogens (AIEgens): Promoting the Dissymmetry Factor and Emission Efficiency Synchronously. ACS Materials Lett. 2020, 2 (5), 505-510.

30. Shi, Y.; Yin, G.; Yan, Z.; Sang, P.; Wang, M.; Brzozowski, R.; Eswara, P.; Wojtas, L.; Zheng, Y.; Li, X.; Cai, J., Helical Sulfono$\gamma$-AApeptides with Aggregation-Induced Emission and Circularly Polarized Luminescence. J. Am. Chem. Soc. 2019, 141 (32), 12697-12706.

31. Li, H.; Li, B. S.; Tang, B. Z., Molecular Design, Circularly Polarized Luminescence, and Helical Self-Assembly of Chiral Aggregation-Induced Emission Molecules. Chem. Asian J. 2019, 14 (6), 674-688.

32. Li, L.; Chen, M.; Zhang, H.; Nie, H.; Sun, J. Z.; Qin, A.; Tang, B. Z., Influence of the Number and Substitution Position of Phenyl Groups on the Aggregation-Enhanced Emission of BenzeneCored Luminogens. Chem. Commun. 2015, 51 (23), 4830-4833.

33. Jasti, R.; Bhattacharjee, J.; Neaton, J. B.; Bertozzi, C. R., Synthesis, Characterization, and Theory of [9]-, [12]-, and [18]Cycloparaphenylene: Carbon Nanohoop Structures. J. Am. Chem. Soc. 2008, 130 (52), 17646-17647.

34. Takaba, H.; Omachi, H.; Yamamoto, Y.; Bouffard, J.; Itami, K., Selective Synthesis of [12] Cycloparaphenylene. Angew. Chem. Int. Ed. 2009, 48 (33), 6112-6116.

35. Golder, M. R.; Jasti, R., Syntheses of the Smallest Carbon Nanohoops and the Emergence of Unique Physical Phenomena. Acc. Chem. Res. 2015, 48 (3), 557-566.

36. Omachi, H.; Segawa, Y.; Itami, K., Synthesis of Cycloparaphenylenes and Related Carbon Nanorings: A Step toward the Controlled Synthesis of Carbon Nanotubes. Acc. Chem. Res. 2012, 45 (8), 1378-1389.

37. Zhang, X. Y.; Shi, H.; Zhuang, G. L.; Wang, S. D.; Wang, J. Y.; Yang, S. F.; Shao, X.; Du, P. W., A Highly Strained All-Phenylene Conjoined Bismacrocycle. Angew. Chem. Int. Ed. 2021, 60 (32), 17368-17372.

38. Darzi, E. R.; Jasti, R., The Dynamic, Size-Dependent Properties of [5]-[12]Cycloparaphenylenes. Chem. Soc. Rev. 2015, 44 (18), 6401-6410.

39. Kido, J.; Kimura, M.; Nagai, K., Multilayer White Light-Emitting Rrganic Electroluminescent Device. Science 1995, 267 (5202), 1332-1334.

40. Lee, S. Y.; Yasuda, T.; Yang, Y. S.; Zhang, Q.; Adachi, C., Luminous Butterflies: Efficient Exciton Harvesting by Benzophenone Derivatives for Full-Color Delayed Fluorescence OLEDs. Angew. Chem. Int. Ed. 2014, 53 (25), 6402-6406. 
41. Rao, K. V.; Datta, K. K. R.; Eswaramoorthy, M.; George, S. J., Highly Pure Solid-State White-Light Emission from SolutionProcessable Soft-Hybrids. Adv. Mater. 2013, 25 (12), 1713-1718.

42. He, Z.; Zhao, W.; Lam, J. W. Y.; Peng, Q.; Ma, H.; Liang, G.; Shuai, Z.; Tang, B. Z., White Light Emission from a Single Organic Molecule with Dual Phosphorescence at Room Temperature. Nat. Commun. 2017, 8 (1), 1-8.

43. Xie, Z.; Chen, C.; Xu, S.; Li, J.; Zhang, Y.; Liu, S.; Xu, J.; Chi, Z., White-Light Emission Strategy of a Single Organic Compound with Aggregation-Induced Emission and Delayed Fluorescence Properties. Angew. Chem. Int. Ed. 2015, 54 (24), 7181-7184.

44. Yang, Q. Y.; Lehn, J. M., Bright White-Light Emission from a Single Organic Compound in the Solid State. Angew. Chem. Int. Ed. 2014, 53 (18), 4572-4577.
45. Tanaka, H.; Inoue, Y.; Mori, T., Circularly Polarized Luminescence and Circular Dichroisms in Small Organic Molecules: Correlation Between Excitation and Emission Dissymmetry Factors. ChemPhotoChem 2018, 2 (5), 386-402.

46. Song, F.; Zhao, Z.; Liu, Z.; Lam, J. W. Y.; Tang, B. Z., Circularly Polarized Luminescence from AIEgens. J. Mater. Chem. C 2020, 8 (10), 3284-3301.

47. Sánchez-Carnerero, E. M.; Agarrabeitia, A. R.; Moreno, F.; Maroto, B. L.; Muller, G.; Ortiz, M. J.; de la Moya, S., Circularly Polarized Iuminescence from Simple Organic Molecules. Chem. Eur. J. 2015, 21 (39), 13488-13500. 
Insert Table of Contents artwork here

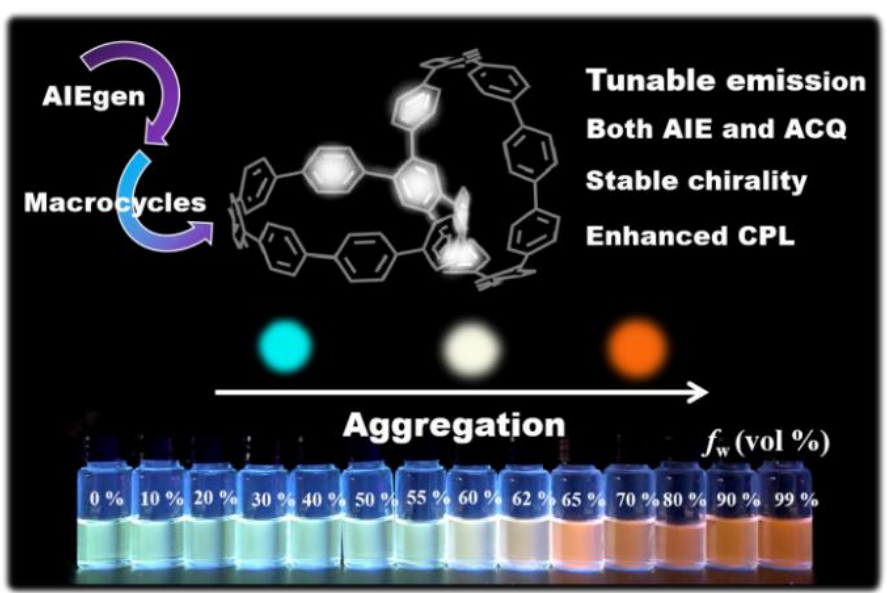

